\title{
FastTomo: A SerialEM Script for Collecting Electron Tomography Data
}

\author{
Albert $\mathrm{Xu}, \mathrm{Chen} \mathrm{Xu}^{\dagger}$ \\ Department of Biochemistry and Molecular Pharmacology \& Cryo-EM Core Facility \\ University of Massachusetts Medical School \\ Email: albert.t.xu@gmail.com, ${ }^{\dagger}$ Chen.Xu@umassmed.edu
}

\begin{abstract}
FastTomo is a SerialEM script for collecting tilted specimen images in transmission electron microscopes to be further used in tomographic reconstruction. It achieves a speedup over conventional tracking methods by minimizing the usage of off-target tracking shots, and instead applies proportional control to the specimen images. Movement in the $Z$ coordinate is estimated prior to each tilt series in a separate calibration routine. Overall, this method is fast and reliable when the field of view is at least $1 \mathrm{um}$, and can tolerate minor errors in setting eucentric height. The implemented tilt series schemes include the unidirectional, bidirectional, and dose-symmetric schemes.

Index Terms-electron tomography, cryoET, eucentricity, proportional control, pre-calibration
\end{abstract}

\section{INTRODUCTION}

Electron tomography is a method of reconstructing a 3D volume from 2D projected images taken at various angles by an electron microscope. In order to successfully collect a series of images, two major problems must be addressed. The first problem is that the sample will move out of view of the camera, so the specimen must be tracked in $\mathrm{X}$ and $\mathrm{Y}$ coordinates. The other problem is that the specimen will move out of focus, and therefore deviation in the $\mathrm{Z}$ coordinate must also be accounted for.

We show that eucentric height is a factor in both of these problems. We address $\mathrm{Z}$ movement using the pre-calibration method described in [1]. We track $\mathrm{X}$ and $\mathrm{Y}$ movement using proportional control - i.e. aligning each image to a previous image taken at an adjacent angle, and adjusting the microscope's image shift deflectors to bring the specimen back to the center of view.

Eucentric height for a given specimen is defined as the $\mathrm{Z}$ coordinate of the stage where the center of rotation of the specimen coincides with the tilt axis of the stage. When the specimen is at this height, the stage is said to be at optimal eucentricity. This height minimizes the total lateral movement when the specimen is tilted over a range of angles. However, even when the specimen is at eucentric height, we show that movement in $\mathrm{X}, \mathrm{Y}$, and $\mathrm{Z}$ is still problematic.

Using proportional control to track $\mathrm{X}$ and $\mathrm{Y}$ movement at low and medium magnification is reliable due to a greater signal-to-noise ratio compared to high magnification, and therefore has a lower chance of observing faulty image correlations. The method is simple; at every tilt angle the specimen

\footnotetext{
$\dagger_{\text {corresponding author }}$
}

image is correlated with a previous image taken at an angle closest to the current angle. For small step sizes $(<4 \mathrm{deg})$ and with an appropriate stretch of one image, the image features are nearly the same and will not affect correlation. The displacement between these two images is used to update the microscope's image shift action using SerialEM's AlignTo function. Overall, this method is fast and has negligible error. Most notably, time is saved by avoiding off-target tracking shots, which are more robust but slower.

$\mathrm{Z}$ movement is estimated by fitting calibrated data to a model. Focus measurements are taken at a nearby location along the tilt axis to estimate a sinusoid model of the $\mathrm{Z}$ coordinate as a function of tilt angle. In practice, we find that calibrating every time before collecting a tilt series is a good trade-off between runtime and accuracy compared to methods which calibrate less frequently. As mentioned in [1], errors in setting eucentric height will vary each time a eucentricity routine is performed, which can lead to poor estimates of $\mathrm{Z}$ movement. Calibrating at each specimen location avoids this problem.

\section{Stage Behavior at Different Heights}

This section illustrates how the stage moves in the $\mathrm{Y}$ and $\mathrm{Z}$ coordinates for several initial heights around eucentric height, and shows the effects of perturbation error when this height is not optimal.

\section{A. Movement in $Z$}

Figure 1 shows the behavior of the stage in the $\mathrm{Z}$ coordinate for several height offsets from a nominal eucentric height. The procedure to obtain the data is as follows:

1) SerialEM's fine eucentricity routine was performed to move the stage to a nominal eucentric height

2) A set of focus measurements were acquired in a bidirectional tilt series scheme, using image shift to keep the specimen point centered

3) The stage was lowered by 2 um

4) Focus measurements were similarly acquired in increments of 0.5 um for 9 iterations

The pre-calibration method is used to predict the $\mathrm{Z}$ coordinate. The method takes a sample of 5 focus measurements (at $-40,-22,0,22,40 \mathrm{deg}$ ) at a nearby area along the tilt axis, and does a least squares fit to the following sinusoid model

$$
y=b_{1} \sin (\theta+\phi)+b_{0}
$$




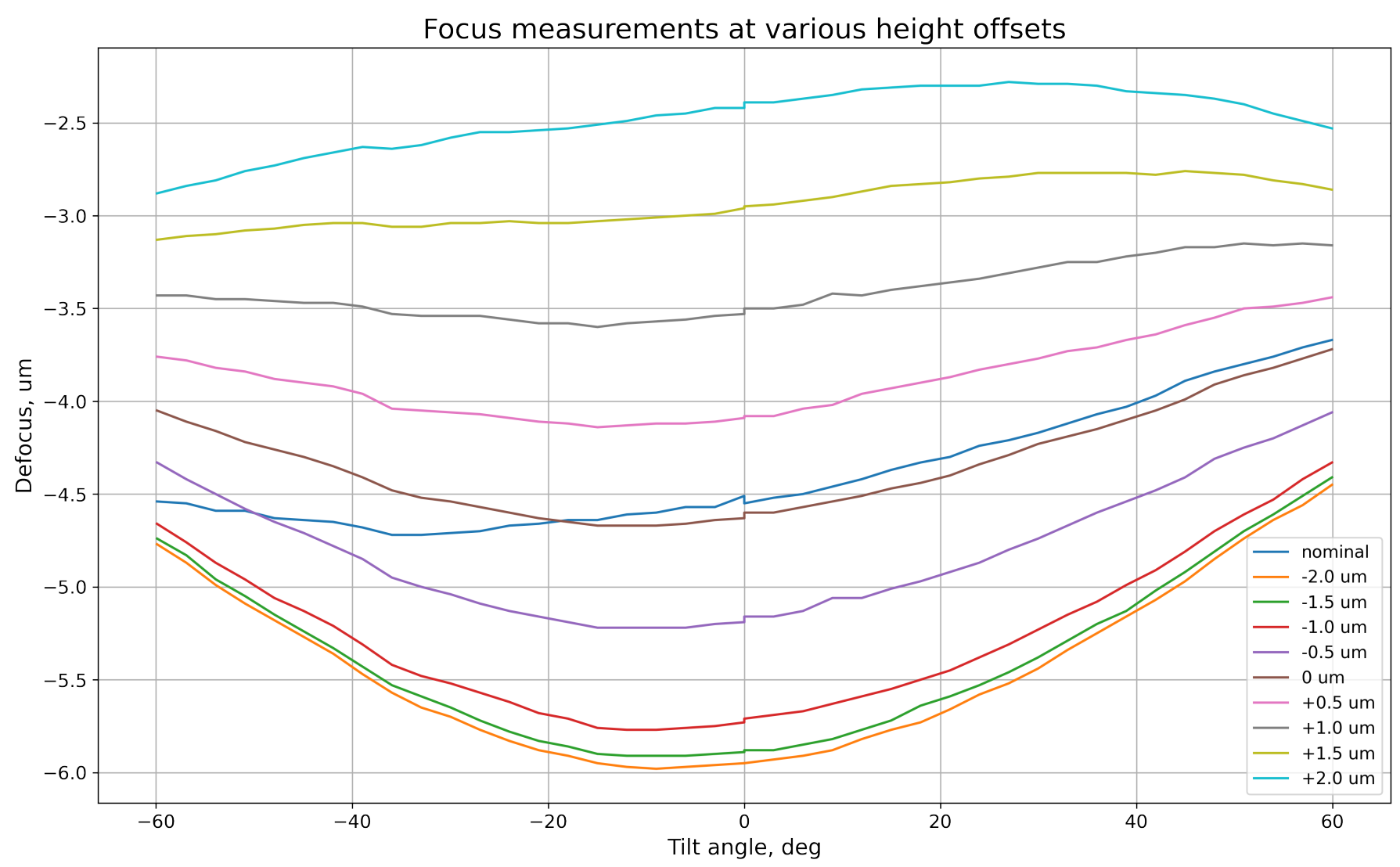

Fig. 1. Focus measurements at different height offsets from a nominal eucentric height. Each tilt series was collected in a bidirectional scheme (angular range: 0 to $\pm 60 \mathrm{deg}$; step size: $3 \mathrm{deg}$ ). The series for the nominal eucentric height is inconsistent with the others, which shows that the behavior varies across routines that adjust stage height. The first two series at -2 um \& -1.5 um offsets are inconsistently spaced due to stage backlash.

with parameters $\left(b_{1}, b_{0}, \phi\right)$, where $y$ is the measured focus at the angle $\theta$. This model encapsulates the rotation of an object around an ideal tilt axis, with inital displacements both laterally and in height.

A limitation of the pre-calibration method described above is that the specimen point cannot be centered due to large feature differences at different views. This means that the sample measurements are taken at different positions. Assuming that the nearby area around the specimen is planar, this modeling error is equal to $h \sin (\theta) \tan (\theta)$, where $h$ is the eucentric height error. For example, the error between the true focus and a sample measurement at 40 degrees and is $0.54 h$.

\section{B. Movement in $Y$}

Figure 2 shows displacement in $\mathrm{Y}$ for several height offsets from a nominal eucentric height. The $\mathrm{Y}$ coordinate in SerialEM's specimen coordinate system is perpendicular to the direction of the tilt axis. The displacements in $\mathrm{Y}$ from 0 degrees were acquired along with the focus measurements from Figure 1, by recording the image shift values that kept the specimen centered in the field of view. Even at near optimal heights, the maximum deviation is significant and requires tracking to ensure that the specimen stays in view.
Movement in $\mathrm{X}$ (parallel to tilt axis) was also recorded but was insignificant and so is not shown.

\section{RESULTS}

This section shows the effects of applying predictions for tracking $\mathrm{Z}$ movement, and a table of total runtimes used for several choices of tilt series parameters.

\section{A. Using predictions for $Z$ movement}

Figure 3 shows the effect of applying the model for predicting $\mathrm{Z}$ movement in an extreme example with particularly suboptimal eucentricity. Compensating for $\mathrm{Z}$ movement limits the maximum deviation to about $1 \mathrm{um}$ from 0 deg tilt.

\section{B. Runtime}

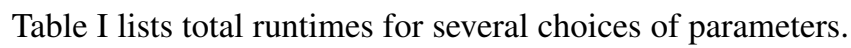
Each tilt series included the $\mathrm{Z}$ calibration routine, which on average takes 70 seconds.

\section{Discussion}

The choice of proportional control as a tracking method has several benefits, namely speed and simplicity. Without the expensive operation of separate tracking shots which alter beam and magnification settings, the majority of time spent in the script is occupied by stage settling and camera recording 
bioRxiv preprint doi: https://doi.org/10.1101/2021.03.16.435675; this version posted March 17, 2021. The copyright holder for this preprint (which was not certified by peer review) is the author/funder, who has granted bioRxiv a license to display the preprint in perpetuity. It is made available under aCC-BY 4.0 International license.

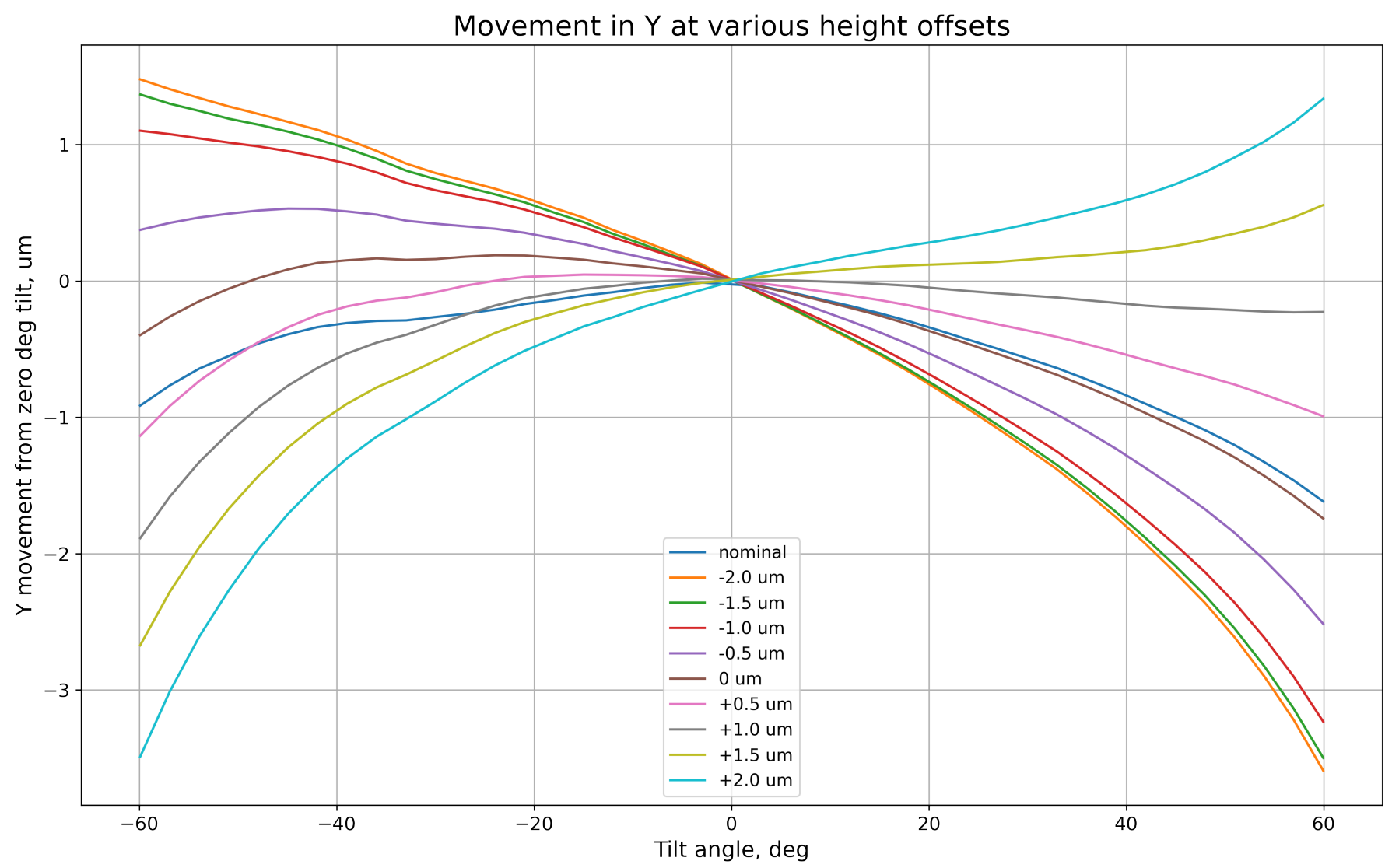

Fig. 2. Y movement at different height offsets from a nominal eucentric height. The minimum deviation from 0 degrees out of all data series is roughly 1 um, which shows that tracking must be done even at eucentric height.

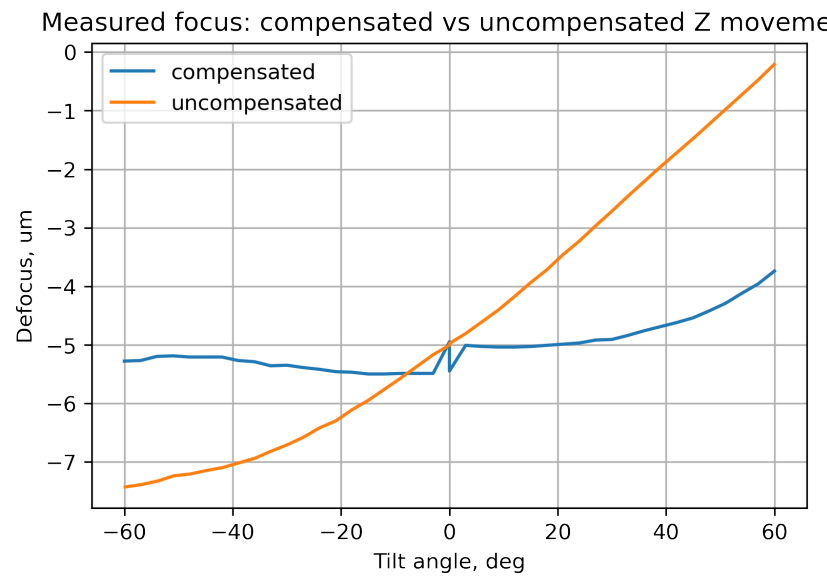

Fig. 3. An extreme case of poor eucentricity. The uncompensated tilt series only tracks the specimen in X \& Y without regarding $\mathrm{Z}$. The compensated tilt series uses FastTomo. The measurements for compensated $\mathrm{Z}$ movement used the same bidirectional scheme as before ( 0 to $-60 \mathrm{deg}, 0$ to $+60 \mathrm{deg}$, 3 deg increments). Note: the uncompensated tilt series used a unidirectional scheme (-60 to $+60 \mathrm{deg}, 3 \mathrm{deg}$ increments), so there is no discontinuity at 0 degrees.

time. This method is very reliable for a sufficiently large field of view of at least 1 um. For higher magnifications,
TABLE I

RUNTIME

\begin{tabular}{|c|c|c|c|}
\hline Tilt series scheme & Angular range & Step size & Runtime \\
\hline Unidirectional & -45 to $+45 \mathrm{deg}$ & $3 \mathrm{deg}$ & $5 \mathrm{~min} 44 \mathrm{~s}$ \\
Unidirectional & -45 to $+45 \mathrm{deg}$ & $2 \mathrm{deg}$ & $7 \mathrm{~min} 8 \mathrm{~s}$ \\
Bidirectional & 0 to $\pm 60 \mathrm{deg}$ & $3 \mathrm{deg}$ & $7 \mathrm{~min} 12 \mathrm{~s}$ \\
Bidirectional & 0 to $\pm 60 \mathrm{deg}$ & $2 \mathrm{deg}$ & $9 \mathrm{~min} 16 \mathrm{~s}$ \\
Dose-symmetric & 0 to $\pm 60 \mathrm{deg}$ & $3 \mathrm{deg}$, groups of 3 & $8 \mathrm{~min} 47 \mathrm{~s}$ \\
Dose-symmetric & 0 to $\pm 60 \mathrm{deg}$ & $2 \mathrm{deg}$, groups of 4 & $11 \mathrm{~min} 4 \mathrm{~s}$ \\
\hline
\end{tabular}

Tested on an FEI Talos Arctica. Exposure time per image was 1.16s using a Gatan K3 camera.

two main problems occur during data collection. Firstly, the displacement between images at successive angles becomes too large relative to the field of view, resulting in insufficient overlapping area and thus failed image correlation. This is especially noticeable when eucentricity is not optimal. Secondly, the lower signal-to-noise ratio results in statistically unreliable image correlations. In the low-dose regime of cryotomography, there is a higher probability of a faulty correlation, at which point the specimen will be lost from view.

Other methods for tracking lateral movement that were considered include: calibrating X \& Y in the same way for Z; iterative polynomial fitting; and PID control - a widely popular 
feedback control algorithm used in industrial applications. In the calibration method, the lateral movement is predicted from a model and applied later. This method can be potentially unreliable because of the same concern for $\mathrm{Z}$ calibration, which is that setting eucentric height has varying error, even at the same stage location. An error in $\mathrm{Z}$ even as small as 0.5 um can have a lateral error of almost 1 um at maximum tilt, i.e. the sample will move out of view. Doing calibrations at every specimen location still did not alleviate this problem and added extra time to the calibration routine. A second method that was tested was iterative polynomial fitting. Unlike the calibration method, this method updates a polynomial fit to image displacements as data is collected, which is then used to make a prediction for the next displacement. This method does better, but still has about 0.5 um error at maximum tilt. Moreover, other considerations need to be made, such as robust regression to outlier data, which is observed on some microscope stages as large jumps at small nonzero angles. Another method that was tested was PID control, which is a historically well-known feedback controller which requires tuning gain parameters for the $\mathrm{P}, \mathrm{I}$, and $\mathrm{D}$ terms. Using very precise tuning, this method does slightly better than calibration and polynomial fitting, but was found to be impractical for the user to tune and thus unstable. The proportional control algorithm described in this paper does not require parameter tuning; in fact, it is a simple case of PID control with unity $\mathrm{P}$ gain and zero I \& D gain. Ultimately, the simplicity and sufficient accuracy of proportional-only control is the reason why it was implemented.

The model for $\mathrm{Z}$ movement was chosen to be a sinusoid instead of a linear equation since the observed measurements in Figure 1 were nonlinear for all heights, including those near eucentric height, and especially for those farther away. Although [2] \& [3] show linear behavior near eucentric height, in practice there is a precision error in setting eucentric height which is purportedly 0.1-0.25 um [1], causing the stage to move nonlinearly. Furthermore, the movement is not necessarily identical at different stage locations, even within the same grid square. Other models such as splines may also be effective, although we find that a sinusoid is accurate enough. In the case that the behavior is largely linear, it can be approximated reasonably well by $\sin$ over half of the tilt range, without the need to add a linear term to the model and risk overfitting.

\section{CONClusion}

In this paper we showed how errors in setting eucentric height affect stage movement, and utilized proportional control and pre-calibration to simplify and speed up electron tomography data collection.

\section{CODE}

The latest version of FastTomo can be found at https: //github.com/alberttxu/FastTomo

\section{ACKNOWLEDGMENT}

We would like to thank David Matronarde for insightful discussion, Jonah Murphy for general comments, and Jun Liu for feedback on user experience.

\section{REFERENCES}

[1] Mastronarde DN. Automated electron microscope tomography using robust prediction of specimen movements. J Struct Biol. 2005 Oct;152(1):36-51. doi: 10.1016/j.jsb.2005.07.007. PMID: 16182563.

[2] Chreifi, G., Chen, S., Metskas, L. A., Kaplan, M., \& Jensen, G. J. (2019). Rapid tilt-series acquisition for electron cryotomography. Journal of Structural Biology, 205(2), 163-169. doi: 10.1016/j.jsb.2018.12.008

[3] Eisenstein, F., Danev, R., \& Pilhofer, M. (2019). Improved applicability and robustness of fast cryo-electron tomography data acquisition. Journal of Structural Biology, 208(2), 107-114. doi: 10.1016/j.jsb.2019.08.006 